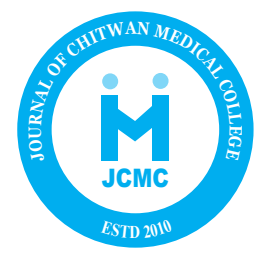

\title{
ROLE OF NUTRACEUTICAL- BRANCHED CHAIN AMINO ACIDS FOR ATTENUATING SKELETAL MUSCLE SORENESS IN POST EXERCISE STATUS AND CENTRAL FATIGUE
} Satish Kumar Deo ${ }^{1 *}$, Akritee Pokharel ${ }^{1}$, Samir Lamichhane ${ }^{1}$, Anish Mudvari ${ }^{1}$

${ }^{1}$ Department of clinical Pharmacology, Maharajgunj Medical Campus, Institute of Medicine, Tribhuvan University, Kathmandu, Nepal.

*Correspondence to: Dr Satish Kumar Deo, Department of clinical Pharmacology, Maharajgunj Medical Campus, Institute of Medicine, Tribhuvan University, Kathmandu, Nepal. Email: satdeo@gmail.com

\begin{abstract}
Implementation of food extract for target medicinal purpose is a genre in medical science called Nutraceutical which is vast and much of it is yet to be explored. Nowadays general population are aware of the importance of exercise in terms of health benefits but the fatigue and delayed onset muscle soreness (DOMS) are limitations that are to be overcome to continue extensive exercises. For this article, the role of Branched chain amino acids (BCAA) for the purpose is studied from various scientific journal. DOMS is due to direct muscle damage following exercise whereas central fatigue is the sensation of fatigue due to neurochemical changes in brain following exercise. In various studies, DOMS and central fatigue were minimized in terms of duration when given to animals and human volunteers. Further research has to be done regarding dosing regimen of BCAA supplementation and also to uncover any adverse effect of the supplements.
\end{abstract}

Key words: Branched chain amino acids, Nutraceuticals, Delayed Onset Muscle Soreness, Central fatigue, Exercise.

\section{INTRODUCTION}

Branched chain amino acid (BCAA) supplementation is an example of nutraceutical. Nutraceuticals are products isolated from food and are generally sold in medicinal forms. They are defined as any food (or part hereof) which provide health benefits including prevention and treatment of disease in addition to the basic nutritional value found in foodstuff. ${ }^{1}$

Muscle soreness is a common setback experienced during endurance exercise evident by the decrease in skeletal muscle strength and function. ${ }^{2}$ Although the mechanism is still unclear, it has been hypothesized that inflammation and muscle damage are keys to its causation. ${ }^{2}$ The muscle action regularly seen can be categorized as eccentric, concentric and isometric, of which eccentric movements bears the most tension. ${ }^{3}$ Muscle soreness and damage is the result of eccentric exercise and is marked by leakage of creatine kinase $(C K)$, lactate dehydrogenase (LDH) and myoglobulins into the circulation that are otherwise found in myocytes. ${ }^{2}$
Endurance exercise induced fatigue can be defined as the failure in sustenance of level of energy required for continuous muscle movement. ${ }^{4}$ This exercise induced fatigue is divided into two types: peripheral fatigue and central fatigue. Even though the cause of fatigue has multifactorial origin, the peripheral type is due to metabolites accumulation, protein breakdown and carbohydrate consumption whereas the central type is said to be due the alteration in central nervous system (CNS) functions which in turn is due to change in neurotransmitter level that confers variation in mood, sensation of effort and tolerance to pain and discomfort. ${ }^{4}$

It is a well-established fact that during exercise, body increases proteolysis and utilizes amino acids (AAs). BCAAs are the ones primarily used as a source of energy in endurance exercise of skeletal muscle. Protein breakdown induced by prolonged exercise is reported to be attenuated by BCAA supplementation. ${ }^{2}$ 


\section{Amino Acids and BCAA}

Protein is one of the major constituents of human body and is also a key component in muscle building. It has a diverse range of functions in our body from muscle building to immunity. A protein is a combination of one or more AAs, a compound containing carboxylic and amine moiety. There are 20 AAs of which 9 are essential amino acids (EAAs) that are not synthesized within our body, and 1 of them is essential only during positive nitrogen balance e.g. during growth. ${ }^{2}$

Three EAAs with diverse physiological and metabolic roles, namely leucine, isoleucine and valine, have some unique properties as compared to the rest of EAAs, are grouped under BCAAs. ${ }^{5}$ As the name suggests, they have branched structure and are predominantly oxidized in skeletal muscles unlike other AAs that are primarily metabolized in liver. BCAAs act centrally and peripherally. Peripherally, BCAAs are thought to be involved in the regulation of protein synthesis and degradation of skeletal muscle. ${ }^{5}$ Centrally, BCAAs compete with aromatic amino acids like tryptophan (a precursor of serotonin, a neurotransmitter) for the same carrier protein and hence have an important role in modulating the level of neurotransmitter.

\section{Types of skeletal muscle}

Skeletal muscle fibers can be classified into slow i.e. type I muscle fibers and fast i.e. type II muscle fibers. Type I fibers are also called red type or slow twitch fiber and they get their name due to the presence of high number of myoglobulins and mitochondria. These muscles are fatigue resistant, highly aerobic with slow speed of contraction, with high capillary density, and small motor unit size. On the other hand, type II fibers are also called white or fast twitch fiber and they get their name due to presence of fewer number of myoglobulins and mitochondria. Type II muscle fibers are further divided into type IIA and type IIB fibers. These muscles are moderate to most fatigable, with medium to low aerobic capacity, fast speed of contraction, have medium to low capillary density and medium to large motor unit size. ${ }^{6}$ Our body has a combination of these two types of muscle fibers. Type I fibers are for endurance exercises such as marathons and cycling, whereas, type II fibers are for short burst exercises such as sprints. ${ }^{3}$

Mechanism of development of delayed-onset

\section{muscle soreness}

Delayed-onset muscle soreness (DOMS) in humans can be described as the discomfort and pain one experiences after exhaustive exercise which occur in a deferred manner. Expressions like stiffness, tenderness or aching are usually used to describe soreness. The stiffness so described are thought to be caused due to perimuscular connective tissue edema rather than due to antagonistic action on muscle. If nature is allowed to take its course, the symptoms of DOMS begin within 24 to 48 hours, ${ }^{7}$ soar around 24 to 72 hours and subside within 5 to 7 days. Various studies have demonstrated tenderness on palpation along with decreased range of motion and an appreciably prolonged loss of strength. ${ }^{3}$ Rise of serum $\mathrm{CK}$, an intramuscular enzyme responsible for sustaining sufficient amount of adenosine triphosphate (ATP) during muscle contraction, is seen with eccentric strenuous exercise. ${ }^{2}$ The presence of CK in serum is believed to be due to increased permeability or breach in muscle cell membrane. ${ }^{3}$

Investigators of experimental studies on eccentric exercise of skeletal muscles have deduced that the early event of muscle injury is mechanical in nature. In an experiment performed on rabbits, histologic examination of tibialis anterior muscle displayed larger, more rounded and lighter stained fibers in comparison to the surrounding tissues after an insult induced by cyclic eccentric exercise. ${ }^{8}$ Further, immunohistochemical studies show breach in the cytoskeleton within the fibers. ${ }^{9}$ These changes were also seen in percutaneous needle biopsy of lateral gastrocnemius muscle taken from humans after distance running and resistance exercises. ${ }^{10}$

Amongst the type of skeletal muscle fibers, type IIB fibers can produce high level of intrinsic force and extrinsic stretch, thus making them vulnerable to injury. ${ }^{11}$ The damage ranges from subtle wavy appearance of the Z-band to the complete distortion of the $A$ or $Z$ band at the ultrastructural level. ${ }^{3}$ Infiltration of circulating monocytes into the damaged muscle cells (which now become macrophages) provides evidence that inflammation has ensued. On this basis, researchers have further hypothesized an association between inflammatory cells infiltration and proteolytic degradation of muscle tissue and there are speculations that the cause of muscle pain is the result of nerve ending stimulation owing to 
the increase in intrafiber pressure and metabolitespotassium ions, nucleotides and inorganic phosphate to name few. ${ }^{3}$ The breach in myocytes following a strenuous exercise facilitates the leakage of metabolites into the blood and contributing to muscle soreness. Also, the increase in plasma granulocyte elastase concentration is attribution of an inflammatory response. ${ }^{12}$

\section{Role of BCAA supplements in management of DOMS}

During exercise, after the exhaustion of available short-term glycogen stores in muscle, BCAAs are utilized as a source of energy. ${ }^{2}$ BCAA catabolism takes place in mitochondria, the initial steps being shared by all BCAAs. The reaction starts with the reversible transamination of BCAA catalyzed by the enzyme branched chain aminotransferase which produces branched chain $\alpha$-ketoacid (BCKA). This is followed by an irreversible oxidative decarboxylation of BCKA catalyzed by the enzyme branched chain $\alpha$-ketoacid dehydrogenase (BCKDH) complex generating coenzyme A (CoA). CoA compounds can enter citric acid cycle as intermediates and can also be used in gluconeogenesis. ${ }^{17}$ During continuous exercise, the deficit supply of BCAAs is fulfilled by muscle breakdown. In many instances despite muscle breakdown, blood BCAAs cannot be maintained at their optimum levels. ${ }^{18}$

There are many experiments which has supported the notion that BCAA supplementation suppresses muscle soreness which is a consequence of muscle protein breakdown. ${ }^{19}$ Increase in plasma Creatine Kinase (CK) (normal range: 60 to $174 \mathrm{IU} / \mathrm{L}$ ) ${ }^{20}$ is the most frequently used parameter to quantify the extent of muscle damage.

In a double blinded crossover study by K. Matsumoto, et al., twelve long distance runners of $20 \pm 1$ years had undergone two intensive training periods each lasting for three days. They were supplemented with either BCAA or an isocaloric placebo drink. The assessment on whole body soreness and fatigue sensation was based on a visual analogue scale method and the extent of muscle damage and inflammation was based on biological marker namely plasma CK, lactate dehydrogenase (LDH) and granulocyte elastase (GEL) was obtained. BCAA supplementation was effective in attenuation of muscle soreness and the difference in biological markers too supported that muscle damage and inflammation was actually less as with BCAA compared to placebo. ${ }^{2}$

In an experiment by Jackman S.R. et al. participants were given either BCAA supplement or a placebo half an hour before commencing exercise, one and half hour after exercise, additional two times during exercise days and four times on 2 subsequent days following the exercise days. In the experiment, concentration of plasma $\mathrm{CK}$ and myoglobin was shown to elevate after exercise and continued to do so during the entire exercise session for both the groups. ${ }^{21}$ The association between muscle soreness and BCAA supplementation was shown not to have significant effect on muscle soreness, serum markers in DOMS induced by eccentric exercise.

In another experiment by Shimomura et al. participants were given BCAA supplementation with powdered green tea and artificial sweetener containing aspartame in water consumed every day during the exercise session. The assessment was done on the Visual analogue scale that showed soreness of muscle in both the groups on the second and third day of the session but the BCAA supplemented group rated the level of soreness much lower than those of the placebo group, thus suggesting the positive effect of BCAA supplements as compared to placebo in exercising muscles. ${ }^{22}$

In another similar experiment, the effect of overreaching exercise in participants was observed for 3 weeks during which they were given Nutrition build II capsules containing BCAA $1800 \mathrm{mg}$ leucine, $750 \mathrm{mg}$ isoleucine, $750 \mathrm{mg}$ valine and, $2000 \mathrm{mg}$ glutamine, 6pills in the morning and 6 pills in the evening. It was a four week session which consisted of resistance training regimen. On the second and fourth day of training and 36 hours after the training session for the day serum CK was collected. In the samples, concentration of respective parameters turned out to be significantly lower for the group on BCAA supplementation. ${ }^{23}$ Thus supporting the claim that BCAA decreases muscle damage in comparison to placebo.

\section{Mechanism of development of Central Fatigue}

Exercise induced fatigue is a complex phenomenon which is attributed by multiple factors such as 
dopamine, serotonin, amount of oxygen and metabolites concentration. ${ }^{4}$

Exercise induced fatigue is mediated by many neurotransmitters, where successor of tryptophan, serotonin level in brain plays a key role. At normal circumstances, level of free tryptophan in plasma determines the extent of entry into the brain. In the peripheral blood, large portion of tryptophan is found loosely bound to albumin. During exercise, mobilization of Free fatty acids(FFA) displaces tryptophan from albumin increasing the free fraction tryptophan in plasma. ${ }^{4}$ This consequently increases tryptophan uptake across blood brain barrier. ${ }^{5}$ Since BCAAs compete with aromatic amino acids such as tryptophan, phenylalanine for carriers in the brain, diet enriched with BCAAs reduces fatigue by hindering tryptophan uptake in brain and making BCAAs favorable for muscle endurance exercisers. ${ }^{5}$ As serotonin cannot cross the Blood brain barrier, it has to be synthesized within the brain. At normal circumstances, level of free tryptophan in plasma and cerebral blood flow determines the extent of entry of this amino acid into the brain.

Since tryptophan is a precursor of serotonin, entry of unusual amount of tryptophan into brain results in raise in serotonin synthesis. Consequently, central fatigue syndrome ensues, as it is being one of effects of raise in serotonin level in brain. ${ }^{5}$

\section{Role of BCAA supplements in the management of Central Fatigue}

Serotonin (5HT 2A) is mainly seen in cortex, hippocampus, facial motor neurons and Serotonin (5HT2C) is widely distributed throughout the CNS. These subtypes are responsible for mood regulation, hallucinogenic activity and energy balance. ${ }^{4}$

This has been proven in a rat experiment, where ingestion of BCAA solution by rats hindered the ascent of serotonin level. ${ }^{13}$

In an experiment comprising male volunteers competing either marathon or a $30 \mathrm{~km}$ cross country race, both physical and mental performance were evaluated which indicated enhancement in both the parameters in BCAA supplemented participants prior to exercise. However, the experimenter also mentioned that the improved exercise performance was observed only in participants completing the marathon beyond 3 hours and 30 minutes. ${ }^{14}$.

In an experiment by Mittleman et al. BCAA supplement versus polydextrose placebo, the participant comprised of both gender at temperature 34.4 . The increment of $14 \%$ in ability to perform $40 \%$ maximal oxygen uptake exercise was seen in participants under BCAA supplementation as compared to a placebo group. The researcher inferred that the BCAA supplementation essentially reduced tryptophan entry into the central nervous system consequently decreasing the amount of fatigue mediated by serotonin. ${ }^{15}$ However results in subsequent experiments failed to support an effect of BCAA supplementation on exercise capacity when similar experiment was performed at higher temperature. ${ }^{16}$

\section{Body requirement and source of BCAAs}

The daily requirement of BCAAs in our body is $\sim 40 \%$ of the total recommended daily allowance (RDA) of the EAAs. The RDA for leucine is approximately $40 \mathrm{mg} / \mathrm{kg}$ body weight and for isoleucine and valine is $10-30 \mathrm{mg} / \mathrm{kg}$ body weight. The requirement can increase up to 25 times during exercise. BCAAs are naturally found ubiquitously in our food, few examples are whey and milk proteins, chicken, fish, beef, egg, soy protein, baked beans, lima beans, chickpeas, lentil, brown rice, whole wheat, corn, almond, brazil nut, cashew nut, and pumpkin seeds. ${ }^{24}$

\section{CONCLUSION}

Branched chain amino acid(BCAA) supplements in exercising muscle definitely has beneficial effect in the attenuation of the muscle soreness and central fatigue syndrome especially in shortening the duration of the symptoms which probably helps in better endurance and performance of exercise. Even though these symptoms are multifactorial in origin and proper dosing of BCAA is yet to be formulated, these findings opens up new prospects for better understanding of the role and uniqueness of BCAA.

\section{REFERENCES}

1. Meštrović DT. Nutraceutical Industry. 2015 [cited 2016 06-09-22016].

2. Matsumoto $K$, et al. Branched-chain amino acid supplementation attenuates muscle soreness,

JCMC/ Vol 7/ No. 2/ Issue 20/ Apr-Jun, 2017 
muscle damage and inflammation during an intensive training program. Journal of Sports Medicine and Physical Fitness 2009;49(4): 424.

3. Lieber RL, Fridén J. Morphologic and Mechanical Basis of Delayed-Onset Muscle Soreness. Journal of the American Academy of Orthopaedic Surgeons 2002;10(1): 67-73.

4. Meeusen R., et al. Central fatigue. Sports Medicine 2006;36(10):881-909.

5. Monirujjaman M, Ferdouse A. Metabolic and physiological roles of branched-chain amino acids. Advances in Molecular Biology 2014.

6. BuckwalterJA, EinhornTA, SimonSR. Orthopaedic basic science: biology and biomechanics of the musculoskeletal system. Vol. 1. 2000: Amer Academy of Orthopaedic.

7. Armstrong R. Muscle damage and endurance events. Sports medicine 1986;3(5):370-381.

8. Lieber RL, Friden J. Muscle damage is not a function of muscle force but active muscle strain. Journal of Applied Physiology 1993;74(2):520-526.

9. Lieber RL, Thornell LE, Friden J. Muscle cytoskeletal disruption occurs within the first 15 min of cyclic eccentric contraction. Journal of Applied Physiology 1996;80(1):278-284.

10. Warhol $\mathrm{M}$, et al., Skeletal muscle injury and repair in marathon runners after competition. The American journal of pathology 1985;118(2):331.

11. Garrett W, Califf J, Bassett F. Histochemical correlates of hamstring injuries. The American journal of sports medicine 1984;12(2):98-103.

12. Fritz $\mathrm{H}$, et al. Granulocyte proteinases as maediators of unspecific proteolysis in inflammation. 1984.

13. Smriga $M$, et al. Preference for a solution of branched-chain amino acids plus glutamine and arginine correlates with free running activity in rats: involvement of serotonergic-dependent processes of lateral hypothalamus. Nutritional neuroscience 2002;5(3):189.

14. Blomstrand $E$, et al. Administration of branchedchain amino acids during sustained exercise- effects on performance and on plasma concentration of some amino acids. European journal of applied physiology and occupational physiology 1991;63(2):83-88.

15. Mittleman KD, Ricci MR, Bailey SP> Branchedchain amino acids prolong exercise during heat stress in men and women. Medicine and science in sports and exercise 1998;30(1):83-91.

16. Cheuvront SN, et al. Branched-chain amino acid supplementation and human performance when hypohydrated in the heat. Journal of Applied Physiology 2004;97(4):1275-1282.

17. Shimomura $Y$, et al. Exercise promotes BCAA catabolism: effects of BCAA supplementation on skeletal muscle during exercise. The Journal of nutrition 2004;134(6):1583S-1587S.

18. Ohtani M, Sugita M, Maruyama K. Amino acid mixture improves training efficiency in athletes. The Journal of nutrition 2006;136(2):538S-543S.

19. Armstrong R. Mechanisms of exercise-induced delayed onset muscular soreness: a brief review. Medicine and science in sports and exercise 1984;16(6):529-538.

20. Armstrong AW, Golan DE. Pharmacology of hemostasis and thrombosis. Principles of Pharmacology: The Pathophysiologic Basis of Drug Therapy. 3rd ed. Philadelphia: Lippincott Williams \& Wilkins, 2011: 372-400.

21. Jackma, SR, et al. Branched-chain amino acid ingestion can ameliorate soreness from eccentric exercise. Medicine and science in sports and exercise 2010;42(5): 962-970.

22. Shimomura $Y$, et al. Nutraceutical effects of branched-chain amino acids on skeletal muscle. The Journal of nutrition, 2006;136(2):529S-532S.

23. Sharp CP, Pearson DR. Amino acid supplements and recovery from high-intensity resistance training. The Journal of Strength \& Conditioning Research 2010;24(4):1125-1130.

24. Sowers S. A primer on branched chain amino acids. Literature Education Series On Dietary Supplements 2009 [cited 2016 08-06-2016]. 